\title{
BMJ Open Optimising the quality of antibiotic prescribing in out-of-hours primary care in Belgium: a study protocol for an action research project
}

\author{
Annelies Colliers, ${ }^{1}$ Samuel Coenen, ${ }^{1,2,3}$ Hilde Philips, ${ }^{1}$ Roy Remmen, ${ }^{1}$ \\ Sibyl Anthierens ${ }^{1}$
}

To cite: Colliers A, Coenen S, Philips $\mathrm{H}$, et al. Optimising the quality of antibiotic prescribing in out-of-hours primary care in Belgium: a study protocol for an action research project. BMJ Open 2017;7:e017522. doi:10.1136/ bmjopen-2017-017522

- Prepublication history for this paper is available online. To view these files, please visit the journal online (http://dx.doi org/10.1136/bmjopen-2017017522).

$\mathrm{AC}$ and SC contributed equally.

Received 28 April 2017

Revised 14 July 2017

Accepted 4 August 2017

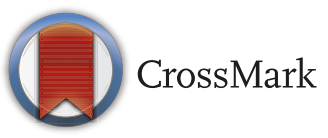

${ }^{1}$ Department of Primary and Interdisciplinary Care (ELIZA)

- Centre for General Practice,

Faculty of Medicine and Health

Sciences, University of Antwerp, Universiteitsplein 1, B-2610 Antwerp, Belgium

${ }^{2}$ Vaccine \& Infectious Disease Institute (VAXINFECTIO),

Faculty of Medicine and Health Sciences, University of Antwerp, Universiteitsplein 1, B-2610

Antwerp, Belgium

${ }^{3}$ Department of Epidemiology and Social Medicine (ESOC),

Faculty of Medicine and Health Sciences, University of Antwerp, Universiteitsplein 1, B-2610 Antwerp, Belgium

Correspondence to Dr Annelies Colliers; annelies.colliers@uantwerpen. be

\section{ABSTRACT}

Introduction Antimicrobial resistance is a major public health threat driven by inappropriate antibiotic use, mainly in general practice and for respiratory tract infections. In Belgium, the quality of general practitioners' (GPS) antibiotic prescribing is low. To improve antibiotic use, we need a better understanding of this quality problem and corresponding interventions. A general practitioners cooperative (GPC) for out-of-hours $(\mathrm{OOH})$ care presents a unique opportunity to reach a large group of GPs and work on quality improvement. Participatory action research (PAR) is a bottom-up approach that focuses on implementing change into daily practice and has the potential to empower practitioners to produce their own solutions to optimise their antibiotic prescribing.

Methods This PAR study to improve antibiotic prescribing quality in $\mathrm{OOH}$ care uses a mixed methods approach. In a first exploratory phase, we will develop a partnership with a GPC and map the existing barriers and opportunities. In a second phase, we will focus on facilitating change and implementing interventions through PDSA (Plan-DoStudy-Act) cycles. In a third phase, antibiotic prescribing quality outside and antibiotic use during office hours will be evaluated. Equally important are the process evaluation and theory building on improving antibiotic prescribing. Ethics The study protocol was approved by the Ethics Committee of the Antwerp University Hospital/University of Antwerp. PAR unfolds in response to the needs and issues of the stakeholders, therefore new ethics approval will be obtained at each new stage of the research.

Dissemination Interventions to improve antibiotic prescribing are needed now more than ever and outcomes will be highly relevant for GPCs, GPs in daily practice, national policymakers and the international scientific community.

Trial registration number NCT03082521; Pre-results.

\section{INTRODUCTION}

Antimicrobial resistance threatens public health worldwide and ranks high on the economic, political and research agendas. ${ }^{1-3}$ The major driver of resistance is inappropriate antibiotic use by humans, the highest proportion of which being prescribed in

\section{Strengths and limitations of this study}

- The participatory action research (PAR) approach is a bottom-up, democratic approach with the active participation of different stakeholders that simultaneously creates scientific and social knowledge with the ultimate goal of change in daily practice.

- The evidence of PAR in changing antibiotic prescribing behaviour is still limited, and has not yet explicitly been done in out-of-hours $(\mathrm{OOH})$ primary care.

- Working within the setting of $\mathrm{OOH}$ primary care offers the potential to reach a large group of general practitioners (GPs) with possible spillover effect to daily practice.

- It might be a challenge to keep the different stakeholders equally involved, committed to the project and convinced of the need to change.

- The quality of the recording of diagnosis and treatment in an electronic medical health record by the GPs could influence the quantitative data on antibiotic prescribing.

ambulatory care, that is, by general practitioners (GPs) for respiratory infections, the most common reason for encounter both during and outside office hours. ${ }^{4}$ Overconsumption and use of broad-spectrum antibiotics are identified as the main quality problems. ${ }^{5}$ To reduce unnecessary antibiotic prescribing in primary care, a variety of interventions were implemented and studied worldwide, with varying success. ${ }^{6-10}$

For nearly two decades, Belgium-like many other countries-has been investing in improving outpatient antibiotic prescribing using national public campaigns ${ }^{11}$ as well as interventions for professionals, including guidelines $^{12}$ and individual antibiotic prescribing feedback. ${ }^{13}$ The national public campaigns have been associated with dramatic decreases in both outpatient antibiotic use 
and antimicrobial resistance since the first campaign in the 2000-2001 winter, but since 2007 outpatient antibiotic use in Belgium is stable, but still twice the level of Sweden and the Netherlands. ${ }^{14}$ Meanwhile, the proportion of antibiotics not recommended as first choice is unacceptably high with a 1:1 ratio of amoxicillin to amoxicillin-clavulanate and $10 \%$ of total outpatient antibiotic use being quinolones, a situation not easily understood nor tackled. ${ }^{15-17}$ Research using well-established disease-specific antibiotic prescribing quality indicators (APQI) revealed low quality of antibiotic prescribing in primary care in Flanders (Northern part of Belgium), especially for respiratory tract infections (RTI), both during and outside office hours. ${ }^{518}$

Meanwhile, the ongoing establishment of general practitioner cooperatives (GPCs) represents one of the most important developments for primary healthcare in Flanders, Belgium and Europe. ${ }^{19}$ In Flanders, about $50 \%$ of residents live in an area covered by a GPC. GPs of that specific region are obliged to participate in this rotation system of being on call during out-of-hours $(\mathrm{OOH})$ in the GPC. These GPCs present a unique opportunity for research and quality improvement as they provide access to large groups of GPs working on one site to care for the same patient population with the same administrative and logistic support, including uniform and mandatory registration of all care episodes in the same electronic medical health record. Moreover, there is a possible spillover effect of any quality improvement in the GPC to primary care during office hours in their respective practices. Until now, detailed data on the quantity and quality of antibiotic prescribing in $\mathrm{OOH}$ care in Belgium, like research on antibiotic prescribing in $\mathrm{OOH}$ care in Belgium, are scarce ${ }^{20}$ Research in Denmark showed high antibiotic prescribing in $\mathrm{OOH}$ care, while in the Netherlands it showed slightly better prescribing quality than during office hours. ${ }^{21} 22$ Both are low-prescribing countries. In this project, interventions will be targeted at 170 GPs of one GPC. Antibiotic prescribing data of three neighbouring GPCs will be available as well to get more detailed insight in prescribing habits in Belgian $\mathrm{OOH}$ care.

'Research that produces nothing but books will not suffice', stated by Lewin, grounding father of action research, remains one of the defining quotes about participatory action research (PAR) ${ }^{23}$ But it is more than true for the battle against antibiotic resistance. In the scientific literature, we can find that several interventions have been proven effective, but it still remains a big societal challenge to implement these interventions and effective change in real practice.

PAR takes into account the facilitators and barriers to the uptake of findings in traditional quantitative research. ${ }^{24}$ It is working with people, rather than doing research on them. ${ }^{25}$ This approach systematically analyses and accounts for the many contextual, cultural and behavioural factors involved in local antimicrobial prescribing, to optimise intervention effectiveness. ${ }^{26}$
PAR works through an iterative process of planning, action and reflection always in close collaboration with the relevant stakeholders. To date, PAR has been used in the acute care setting for hospital inpatients and the long-term care setting of nursing homes and residential care to improve antibiotic use. ${ }^{26}$ So far, it has not yet been explicitly used to improve antibiotic prescribing in $\mathrm{OOH}$ primary care. The effectiveness of any intervention on antibiotic prescribing depends on the particular prescribing behaviour of the physicians and the barriers to change in the particular community, ${ }^{8}$ and this is what PAR takes into account. Although the evidence of PAR in this field is still limited, it is a promising approach to optimise antibiotic prescribing behaviour in the setting of $\mathrm{OOH}$ primary care.

Therefore, in this study we will use the PAR approach to improve the quality of antibiotic prescribing for acute infections in primary care. The goal is to co-create and set up interventions together with the GPs of a GPC. As outcomes, we will use APQI to assess the quality of antibiotic prescribing at the GPC and antibiotic use data to assess a possible spillover effect from the intervention in $\mathrm{OOH}$ care to office hours. In addition, we will assess the feasibility and acceptability of PAR in this setting. And we will describe what can be learnt from the success factors and barriers of using the PAR approach to improve antibiotic prescribing in $\mathrm{OOH}$ primary care.

\section{METHODS/DESIGN PAR design}

Four typologies in action research were described by Hart and Bond as a continuum. ${ }^{27}$ Those four types are the experimental, organisational, professionalising and empowering types of action research. Our approach is most closely related to the empowering type. This bottom-up approach allows participants to be in control of the process and to develop an understanding of the problem, and next to determine possible solutions. To describe an action research project at the start of the study is not easily done as it is per definition dynamic, adapting to the situation, process driven, influenced by practice and participants, and thus continuously changing. ${ }^{28}$

This study protocol sketches the overall plan of the study, but will be responsive to continuous adaptations to fit the goal set by the researchers and participants (figure 1). The study consists of three phases: exploring, facilitating change and evaluation. The study duration will be approximately 4years and will be named the BAbAR study: Better Antibiotic prescribing through Action Research. Start date is April 1st 2017. In the first year, we plan to complete the first exploring phase, in the second and third years, the facilitating change phase, and in the last year we plan to run a detailed evaluation of the project. 


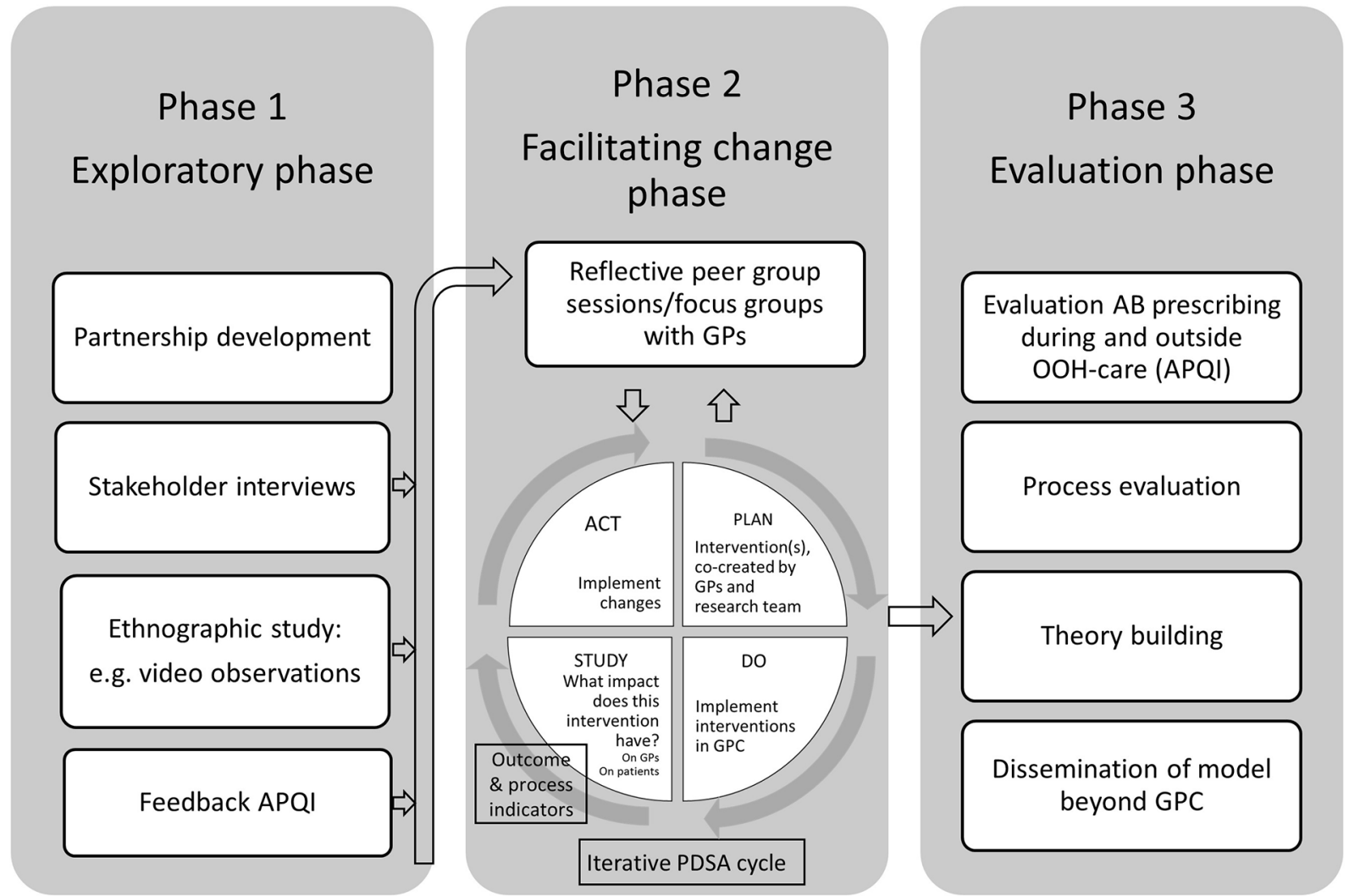

Figure 1 PAR (participatory action research) design of the BAbAR study (Better Antibiotic prescribing through Action Research). AB, antibiotics; APQI, antibiotic prescribing quality indicators; GP, general practitioner; GPC, general practitioner cooperative; OOH, out-of-hours; PDSA, Plan-Do-Study-Act.

\section{Phase 1: exploring}

In the exploratory phase of this research, the focus will first be on partnership development and engaging the GPs with this research project. The success of the PAR approach depends on the willingness of the stakeholders to play an active role. ${ }^{24} 29$

To map the willingness to participation and the need to change, contacts with the GPs of the GPC are planned. Emphasis will be put on the fact that the partnership is non-judgemental, has a reciprocal character and is based on trustworthiness. Developing a positive partnership with the GPs will be essential. A first contact with the board of the GPC has already taken place, and they expressed their willingness to participate in this project.

Next, narrative research will be undertaken to explore how the different stakeholders experience or make sense of antibiotic prescribing within their setting. By better understanding GPs' behaviour and taking into account the different barriers they experience, we will be able to understand their conduct and to develop interventions with better chances of success and bigger support from the GPs. Individual in-depth interviews will be performed with the relevant identified stakeholders (GPs, manager, and so on). A semistructured interview guide will be used. Topics consist on the one hand of the specifics on prescribing antibiotics in $\mathrm{OOH}$ primary care, the perceived need and the receptiveness to change, local antibiotic prescribing culture and habits, and so on, and on the other hand of the willingness to participate in PAR, the degree of commitment in being a coresearcher, the perceived viability of the project, and so on. Purposeful sampling will be performed to obtain a relevant variety in participants that reflects our specific setting (young vs experienced GPs, solo vs group practice during office hours, ethnical background, and so on). Scientific rigour is ensured by using triangulation and member checks involving first the participants in the interviews, and second all other stakeholders (see phase 2). The GPs who have contributed to the interviews will receive a formal analysis report with the summary of the findings and will be asked to deliver feedback (member checking, but also reflecting on it) in a focus group. In a second phase, this will be made available to all GPs of the GPC. Coding of the first three to five interviews will be done by two researchers independently (AC, a GP and SA, a sociologist). The coding framework will then be developed by consensus of these two researchers. Following the independent coding, the initial thematic framework will be compared, and similarities and differences will be discussed and amended to create a set of themes that represents both analyses. This thematic framework will be used for further analysis and if new themes emerge this will be discussed among the research team. The interim analyses will be critically looked at by the other three members of the multidisciplinary research team and will be adapted after their feedback. 
To develop a clear view on the why and when antibiotics are prescribed in $\mathrm{OOH}$ primary care, an ethnographic study will be set up. If consent is given by the GPs, we will use video observations to achieve a better understanding of the context, difficulties, clinical issues, patient-doctor interactions, and so on, during prescribing antibiotics in $\mathrm{OOH}$ primary care. It gives the possibility to gain insight in GPs' habits in their natural, real-world setting. The videos will be observed solely by the research team. Complete anonymity of the GPs and patients will be guaranteed. Using video observations can be a sensitive method for GPs in practice, but a review showed that from an ethical and practical point of view, recording consultations is generally acceptable to both patients and GPs. ${ }^{30} 31$ The data collected through video observations will be analysed on the one hand for research purposes and on the other hand to guide the intervention development. During the interviews of phase 1 , the use of video observations and possible related barriers will be discussed with the participants. During the interviews, we explore the thoughts of the GPs about receiving personal feedback or the possibility to discuss the videos with one of the researchers or with their peers. Another question is asked about the thoughts about using the videos solely as transcripts for research or also for peer education, or even to use them in video format with peers. Hence, the way we will use the video material will depend on the willingness or preference of the participants in our action research.

Because PAR is set up together with the participants having a voice in the design of the study, alternative data collection methods to gain insight in the daily practice of using antibiotics in $\mathrm{OOH}$ primary care can be suggested and subsequently applied. Alternative methods could be a live observation by a member of the research team, a study of the anonymised electronic medical health records, standardised patients, patient interviews, case vignettes, and so on.

Antibiotic prescribing quality at the level of the GPC will be assessed before the start of the PAR using well-established APQI applied before in this setting. ${ }^{5}{ }^{18}$ For the six most common indications for antibiotic prescribing (in descending order: acute bronchitis (ICPC (6) code R78), acute upper RTI (R74), cystitis/other urinary infection (UTI; U71), acute tonsillitis (R76), acute/chronic sinusitis (R75) and acute otitis media (H71)) and for pneumonia (R81) values of three quality indicators will be calculated and fed back $^{5}$ :

A. the percentage of patients prescribed an antibiotic;

B. (A) and receiving the guideline-recommended antibiotic;

C. (A) and receiving quinolones.

The APQI values will be calculated before the start of the PAR in phase 1 and will be used during the PDSA (Plan-Do-Study-Act) cycle(s) of phase 2 as a quantitative indicator of antibiotic prescribing quality improvement in $\mathrm{OOH}$ primary care. In the data analyses, the focus will be on lowering antibiotic prescribing as well as on improving the proportion of receiving a recommended antibiotic and lowering the use of broad-spectrum antibiotics. But, the involved GPs can choose to develop interventions that target all these elements or target only one specific problem. The participants will have their saying in the action process itself, and on how it will be evaluated $^{32}$ so matching interventions and assessments have to be chosen.

We will be able to use data collected through the electronic medical health records. iCAREdata (Improving Care And Research Electronic Data Trust Antwerp; www. icaredata.eu), that is, a research database linking and collecting routine data from all GPCs covering $\mathrm{OOH}$ primary care in Antwerp, allows us to deliver up-to-date feedback and evaluate interventions at GPC level. ${ }^{33} 34$

\section{Phase 2: facilitating change}

Next, PAR will focus on planning and implementing interventions based on the findings in phase 1. Participants are encouraged to be involved in defining the nature of change and the activities to accomplish this change.

In the second phase of our study, qualitative and quantitative results of phase 1 will be fed back to all participants in the PAR. In reflective peer group sessions, the barriers and opportunities will be explored and interventions will be co-designed together with PAR participants taking into account previous work as well as the current scientific knowledge. By reflecting and interacting with each other, a joint strategy grounded in the reality of $\mathrm{OOH}$ primary care practice can originate. Interventions will be delivered and assessed at GPC level, but since the intervention(s) will be co-created with the stakeholders, at this stage it is unclear how the intervention will look like and at who it will be aimed, the individual GP, the patients, both or any other relevant stakeholder or structure. Every GP of the region is on call for several times every year and thus will be exposed to the intervention(s). There are recognised difficulties in measuring effectiveness of interventions in PAR and using PDSA cycles because of the many variables in a complex situation. ${ }^{35}{ }^{36}$ The evaluation of action research therefore is not solely a change intervention, but more a research approach with change and knowledge outcomes, where qualitative findings on context, process and views of participants are a part of.

The feasibility and acceptability of the implemented interventions will be studied from the perspectives of the GPs, and from the perspective of the patients. Process indicators will depend on the type of interventions and implementation strategies chosen by the stakeholders. If, for example, they choose for an internet-based communication skills training such as GRACE INTRO, ${ }^{37-41}$ the number of patient information booklets, which are an integral part of this intervention, distributed to patients could serve as a process indicator. Also the experiences, views, acceptability, and so on, of patients receiving this intervention will be explored and be taken into account into the adaptation of the interventions. At this point, the research group does not have a preference for the type of interventions that will be implemented, but they do 
have the knowledge of existing, appropriate and effective interventions to support the GPs' wishes and needs. We aim to run the PDSA cycle a minimum of two times.

The results of this implementation phase of the study will be reported using the StaRI (Standards for Reporting Implementation Studies) checklist. ${ }^{42}$

\section{Phase 3: evaluation}

In the third phase, the impact of the interventions on the quality of antibiotic prescribing will be evaluated. But equal importance will be given to the evaluation of the process of PAR.

We will evaluate the quality of antibiotic prescribing based on the APQI values for OOH primary care, and also aim to assess any spillover effect to primary care during office hours of improving the quality of antibiotic use outside office hours. The effect on antibiotic use during office hours will be assessed using Intermutualistic Agency (IMA, www.nic-ima.be) data. Hence, the latter outcome measurement will have complete response, and will not interfere with the normal routine of the eligible GPs, allowing a more valid estimate of any spillover effect than databases who recover data from the recording by GPs into their own system. Since IMA data have no link with diagnoses and are data from the dispensing of the medication to the patient, the effect on antibiotic use in general and in relevant subgroups defined by age and gender will be assessed rather than the prescribing by diagnosis. The quality of the IMA data does not depend on the quality of recording by the GPs, in contrast to the $\mathrm{OOH}$ care data. In Belgium, every GP is obliged to be on call in the GPC of his/her own region, meaning that one GPC covers all the GPs of one specific well-defined region.

The process evaluation aims to deepen our understanding of how, why and what was learnt from the project. PAR refers to a range of research methods that emphasise the importance of participants and action. It uses methods that involve iterative processes of reflection and action. ${ }^{43}$ Although most of the PAR methods involve qualitative techniques, increasingly quantitative and mixed methods are used, which we will also combine. The main emphasis however is on the process. The main objectives are to explore, to see whether the process is adequate (are the intervention and outcomes occurring, is there a change in prescribing) and to explain how and why does implementation of the intervention lead to effects (so develop or expand a theory to explain the relationships between concepts and the reason for the change). ${ }^{44}$ The concept of analytic generalisation, by linking our particular findings to theory, allows to apply the research findings in similar contexts, and to other groups and contexts as well. ${ }^{45}$ Dissemination of this model to other GPCs and other GP settings is one of the deliverables.

\section{Setting}

The GPC of the Antwerp city centre covers four districts with more than 187000 inhabitants. More than 50\% of patients have a foreign origin. ${ }^{46}$ There are 170 GPs who are on call in a rotation-based system from Friday evening 19:00 until Monday morning 07:00. They work in shifts of 12 hours. During the day, two GPs are responsible for the consultations at the GPC, while one GP is responsible for home visits. During the night, there is one GP responsible for seeing all patients. There is a secretary for administrative support and a driver for the home visits. The average age of the GPs is 49.3 years old, 78 are men and 92 are women, and 21 of them are GP trainees.

\section{Ethics, registration and dissemination}

Ethics approval for the overall study was obtained from the Ethics Committee of the Antwerp University Hospital/ University of Antwerp (reference number 17/08/089). As each work package of the study develops, amendments might be applied for. The study is registered on clinicaltrials.gov (NCT03082521).

The findings of this project will be discussed with all participants, disseminated at national and international scientific meetings, and published in peer-review journals. In addition, we will discuss both the development and the findings of this project with Belgian Antibiotic Policy Coordination Committee (BAPCOC) to inform future interventions to improve antibiotic use in Belgium.

Ethics approval for data extraction from the electronic medical records for all GPCs in the iCAREdata database was granted by the Ethics Committee of the University of Antwerp/University Hospital Antwerp (12/49/404 and $13 / 34 / 330)$.

To secure the privacy of information about individual patients, a permission for the data collection at the GPCs was obtained from the Committee of Health of the Commission for the Protection of Privacy (No. 14/094n173 on 18 November 2014). A separate application for the data linkage was approved on 28 July 2015 (No. 14/194n133).

An official request to use these specific antibiotic data will be made to the scientific advisory board of iCAREdata.

\section{Researchers and research paradigm}

This PAR project adopts a critical theory approach by critically reflecting on a social system and by applying knowledge from the social sciences. A critical theory approach relies on dialogical methods combining observations and interviewing with approaches to foster conversation and reflection. This reflective dialogic allows the researcher and the participants to question the 'natural' state and challenge the mechanisms for maintenance. ${ }^{25} 47$ The aim is to challenge guiding assumptions and ask people in the organisation to reflect on and question their current practice; not just to describe it but with the ultimate aim to change it.

In PAR, it is important to be clear on the researchers' beliefs and values, which is inseparably linked with the background of the members of the research team. AC 
is a GP and junior researcher at the Centre for General Practice (CHA), Department of Primary and Interdisciplinary Care (ELIZA) of the University of Antwerp, and main researcher of this study. This research will be part of her PhD thesis. She has worked as a GP in this particular GPC of the study until 2015. This makes her role in the study very unique, by having both an insider and an outsider role. SA and SC are the two supervisors of the study. SA is a primary healthcare sociologist at CHA-ELIZA and postdoc researcher with expertise in qualitative social research, with a specific interest in the implementation of antibiotic improvement interventions. SC is associate professor of clinical epidemiology, coheads CHA-ELIZA and associate member of the Vaccine \& Infectious Disease Institute of the University of Antwerp, and chair of the BAPCOC working group coordinating the antibiotic awareness campaigns in Belgium. His research focuses on the multidisciplinary study of infectious diseases, with particular focus on the quality of antibiotic prescribing for RTIs in primary care. $R R$ is professor in general practice, coheads CHA-ELIZA of the University of Antwerp. HP is a postdoc researcher. Like RR, HP is a GP and experienced researcher in the field of $\mathrm{OOH}$ primary care. The team has a strong international network in the field of $\mathrm{OOH}$ care and infectious diseases. The multidisciplinarity of the team is a strong asset. Self-reflective field notes will be kept by the main researcher.

\section{DISCUSSION}

PAR is being used more widely in healthcare settings since the late 1990s to address complex and multifactorial healthcare problems. The use of PAR in development of antimicrobial stewardship programmes is limited and has not yet been tested in the particular setting of the GPC. ${ }^{26}$ It could however be the answer to bridge the research practice gap existing in implementing the changes needed to improve antibiotic prescribing behaviour.

\section{POSSIBLE STRENGTHS AND WEAKNESSES}

In this study, we will reach a large group of GPs with various background (solo practice vs group practice, different age groups, and so on) all working in the same clinical setting. The setting of $\mathrm{OOH}$ primary care has been proven a meaningful and feasible place to work on antibiotic usage. ${ }^{20} 4849$ Hypothetically, a GPC could act as a catalyst for behaviour change in GPs during office hours, forming a suitable and promising setting to implement interventions on behavioural change.

The use of routinely collected data for research purposes and to improve care is gaining more and more interest under the term 'Learning Healthcare systems.${ }^{50-52}$ It offers tremendous possibilities to improve clinical practice. But it also poses challenges such as data quality, security issues, technical support, and so on. ${ }^{50515354}$ In this project, the quality of the data depends on the quality of recording by the GPs in their electronic health record at the GPC. We will monitor the quality of these data closely and critically reflect on the relevance for clinical practice.

Although the focus of this research is the improvement of the quality of antibiotic prescribing, an equally important goal is to see what can be learnt from the process. Meyer stated that the success of action research lays not within the positively demonstrated change, but more within what was learnt from the experience.$^{55}$ Physicians' antibiotic prescribing is influenced by multifactorial elements. Changing their behaviour is a complex task. Trying to understand why and how interventions lead to an effect will be of importance. ${ }^{56}$ Studying the mechanisms underlying the change will be essential to be able to transfer and adapt our approach to other settings and contexts. Reliability is not the goal of PAR. The validity of PAR rests within the movement of action and reflection. The goal is to work on rich, genuine and trustworthy data to strive for transferability to other settings and contexts. Findings of every phase of the research will be discussed and published within the PAR approach and will be provided with rich contextual details to judge relevance for the reader's own context. Generalisation of action research is not empirically based, but theoretically constructed. ${ }^{55}$ Our findings will only be generalisable within our own specific context and situation. The idea is not to seek generalisable data, but generate knowledge. ${ }^{32}$ Critical reflection within the research group and with the stakeholders will continuously feed this knowledge and will sketch the research within a certain context.

Antibiotic rates vary between different GPs. The impact of this intervention on GPs can differ between the prescribers who are adhering to guidelines or the ones who are not. Attention should be paid on involving and motivating these last ones. If consent is given by the GPs, looking at the anonymised individual data is a possibility to generate personal prescribing feedback.

The process of change is a complex and slow process. Implementing new ways and habits in daily practice is a challenging task, and must be widely supported by all stakeholders. The use of (broad-spectrum) antibiotics in primary care in Belgium is among the worst in Europe, despite all efforts to date. ${ }^{14} 17$ We believe that PAR as a bottom-up approach can be the tool to improve the quality of antibiotic prescribing.

Contributors AC, SC, RR, HP and SA contributed to the concept and the design of the study, and drafted and revised the manuscript. All authors have given final approval of the version to be published and they agree to be accountable for all aspects of the work.

Funding The project has been granted a fellowship from the Faculty of Medicine and Health Sciences of the University of Antwerp.

Competing interests None declared.

Ethics approval We attest that we have obtained appropriate permissions and paid any required fees for use of copyright protected materials.

Provenance and peer review Not commissioned; externally peer reviewed. 
Open Access This is an Open Access article distributed in accordance with the Creative Commons Attribution Non Commercial (CC BY-NC 4.0) license, which permits others to distribute, remix, adapt, build upon this work non-commercially, and license their derivative works on different terms, provided the original work is properly cited and the use is non-commercial. See: http://creativecommons.org/ licenses/by-nc/4.0/

(c) Article author(s) (or their employer(s) unless otherwise stated in the text of the article) 2017. All rights reserved. No commercial use is permitted unless otherwise expressly granted.

\section{REFERENCES}

1. Howell L, ed. Global Risks 2013: An Initiative of the Risk Response Network. Geneva: World Economic Forum, 2013.

2. O'Neill J. Tackling a Crisis for the Health and Wealth of Nations: Wellcome Trust and UK Government, 2014.

3. Malhotra-Kumar S, Lammens C, Coenen S, et al. Effect of azithromycin and clarithromycin therapy on pharyngeal carriage of macrolide-resistant streptococci in healthy volunteers: a randomised, double-blind, placebo-controlled study. Lancet 2007;369:482-90

4. Bartholomeeusen S. Morbidity research in primary care, using semiautomatic data collection from electronic medical records in general practices in Flanders. Leuven (Be: Catholic University Leuven, 2008.

5. Adriaenssens N, Coenen S, Tonkin-Crine S, et al. European Surveillance of Antimicrobial Consumption (ESAC): disease-specific quality indicators for outpatient antibiotic prescribing. BMJ Qual Saf 2011;20:764-72.

6. van der Velden AW, Pijpers EJ, Kuyvenhoven MM, et al. Effectiveness of physician-targeted interventions to improve antibiotic use for respiratory tract infections. Br J Gen Pract 2012;62:801-7.

7. Cross EL, Tolfree R, Kipping R. Systematic review of public-targeted communication interventions to improve antibiotic use. J Antimicrob Chemother 2017;72:975-987. epub.

8. Arnold SR, Straus SE. Interventions to improve antibiotic prescribing practices in ambulatory care. Cochrane Database Syst Rev 2005:CD003539.

9. Aabenhus R, Jensen JUS, Jorgensen KJ, et al. Biomarkers as pointof-care tests to guide prescription of antibiotics in patients with acute respiratory infections in primary care. Cochrane Db Syst Rev 2014

10. Coxeter P, Del Mar CB, McGregor L, et al. Interventions to facilitate shared decision making to address antibiotic use for acute respiratory infections in primary care. Cochrane Database Syst Rev 2015:CD010907.

11. Antibiotics. Use them correctly and only when necessary!. http:// www.correctuseantibiotics.be/en

12. Bapcoc. Belgische gids voor anti-infectieuzebehandeling in de ambulante praktijk. http://www.pubmed.be/832250_BW_NL_01_84 IC.pdf

13. Belgian Antibiotic Policy Coordination Committee (BAPCOC). http:// consultativebodies.health.belgium.be/en/advisory-and-consultativebodies/commissions/BAPCOC

14. European Centre for Disease Prevention and Control. Antimicrobial consumption database (ESAC-Net). http://ecdc.europa.eu/ en/healthtopics/antimicrobial-resistance-and-consumption/ antimicrobial-consumption/esac-net-database/Pages/database.aspx

15. Balligand E, Costers M, Van Gastel E. Policy note 2014-2019. Belgian Antibiotic Policy Coordination Committee 2014. Federale Overheidsdienst Volksgezondheid: Veiligheid van de Voedselketen en Leefmilieu(Brussels).

16. Coenen S, Costers M, De Corte S, et al. The first European Antibiotic Awareness Day after a decade of improving outpatient antibiotic use in Belgium. Acta Clin Belg 2008;63:296-300.

17. Versporten A, Bolokhovets G, Ghazaryan L, et al. Antibiotic use in eastern Europe: a cross-national database study in coordination with the WHO Regional Office for Europe. Lancet Infect Dis 2014:14:381-7.

18. Adriaenssens N, Bartholomeeusen S, Ryckebosch P, et al. Quality of antibiotic prescription during office hours and out-of-hours in Flemish primary care, using European quality indicators. Eur J Gen Pract 2014;20:114-20.

19. Philips $H$, Remmen R, Van Royen $P$, et al. What's the effect of the implementation of general practitioner cooperatives on caseload? Prospective intervention study on primary and secondary care. BMC Health Serv Res 2010;10:222.

20. Willems L, Denckens P, Philips H, et al. Can we improve adherence to guidelines for the treatment of lower urinary tract infection? A simple, multifaceted intervention in out-of-hours services. J Antimicrob Chemother 2012;67:2997-3000.
21. Debets VE, Verheij TJ, van der Velden AW, et al. Antibiotic prescribing during office hours and out-of-hours: a comparison of quality and quantity in primary care in the Netherlands. $\mathrm{Br} J$ Gen Pract 2017;67:e178-e186.

22. Huibers L, Moth G, Christensen MB, et al. Antibiotic prescribing patterns in out-of-hours primary care: a population-based descriptive study. Scand J Prim Health Care 2014;32:200-7.

23. Adelman C. Kurt Lewin and the Origins of Action Research. Educational Action Research 1993:1:7-24.

24. Meyer J. Qualitative research in health care. Using qualitative methods in health related action research. BMJ 2000;320:178-81.

25. Reason P, Bradbury H. The SAGE Handbook of Action Research. London: SAGE, 2008.

26. van Buul LW, Sikkens JJ, van Agtmael MA, et al. Participatory action research in antimicrobial stewardship: a novel approach to improving antimicrobial prescribing in hospitals and long-term care facilities. $J$ Antimicrob Chemother 2014;69:1734-41.

27. Hart E, Bond M. Action research for health and social care: a guide to practice. Buckingham: Open University Press, 1995.

28. Fals-Borda O, Action RM. Knowledge: Breaking the Monopoly With Participatory Action Research. London: Intermediate Technology publications, 1991

29. Waterman $\mathrm{H}$, Tillen $\mathrm{D}$, Dickson $\mathrm{R}$, et al. Action research: a systematic review and guidance for assessment. Health Technol Assess 2001;5:iii-157.

30. Themessl-Huber M, Humphris G, Dowell J, et al. Audio-visual recording of patient-GP consultations for research purposes: a literature review on recruiting rates and strategies. Patient Educ Couns 2008;71:157-68.

31. Jepson M, Salisbury C, Ridd MJ, et al. The 'One in a Million' study: creating a database of UK primary care consultations. Brit J Gen Pract 2017.

32. Koshy EKV, Waterman H. Action Research in Healthcare: SAGE Publications Inc, 2011

33. Colliers A, Bartholomeeusen S, Remmen R, et al. Improving Care And Research Electronic Data Trust Antwerp (iCAREdata): a research database of linked data on out-of-hours primary care. BMC Res Notes 2016:9:259.

34. Coenen S, Gielen B, Blommaert A, et al. Appropriate international measures for outpatient antibiotic prescribing and consumption: recommendations from a national data comparison of different measures. J Antimicrob Chemother 2014;69:529-34.

35. Reason P, Bradbury H. The SAGE Handbook of Action Research: Participative Inquiry and Practice. Sage Publications Ltd. 2nd edition, 2008.

36. Reed JE, Card AJ. The problem with Plan-Do-Study-Act cycles. BMJ Qual Saf 2016:25:147-52

37. Tonkin-Crine S, Anthierens S, Hood K, et al. GRACE INTRO/CHAMP consortium. Discrepancies between qualitative and quantitative evaluation of randomised controlled trial results: achieving clarity through mixed methods triangulation. Implement Sci 2016;11:66.

38. Anthierens S, Tonkin-Crine S, Cals JW, et al. Clinicians' views and experiences of interventions to enhance the quality of antibiotic prescribing for acute respiratory tract infections. J Gen Intern Med 2015;30:408-16.

39. Tonkin-Crine S, Anthierens S, Francis NA, et al. Exploring patients' views of primary care consultations with contrasting interventions for acute cough: a six-country European qualitative study. NPJ Prim Care Respir Med 2014;24:14026.

40. Little P, Stuart B, Francis N, et al. Effects of internet-based training on antibiotic prescribing rates for acute respiratory-tract infections: a multinational, cluster, randomised, factorial, controlled trial. Lancet 2013;382:1175-82

41. Anthierens S, Tonkin-Crine S, Douglas E, et al. General practitioners' views on the acceptability and applicability of a web-based intervention to reduce antibiotic prescribing for acute cough in multiple European countries: a qualitative study prior to a randomised trial. BMC Fam Pract 2012;13:101.

42. Pinnock H, Barwick M, Carpenter CR, et al. Standards for Reporting Implementation Studies (StaRI) Statement. BMJ 2017:356:i6795.

43. Peters DH, Adam T, Alonge O, et al. Implementation research: what it is and how to do it. Bmj-Brit Med J 2013:347.

44. Peters DH, Tran N, Adam T. Implementation research in health: a practical guide. Alliance for Health Policy and Systems Research, World Health Organization 2013

45. Rolfe G, Knowledge EN. Oxford: Butterworth Heinemann, 1998.

46. Stad in Cijfers. Stad in Cijfers: zo zoekt u statistieken over de stad op. https://stadincijfers. antwerpen.be/dashboard

47. Kincheloe J, McLaren P. Rethinking critical theory and qualitative research. NK Denzin and YS Lincoln (eds) Handbook of Qualitative Research, 1994:138-57. 
48. de Bont EG, Dinant GJ, Elshout G, et al. An illness-focused interactive booklet to optimise management and medication for childhood fever and infections in out-of-hours primary care: study protocol for a cluster randomised trial. Trials 2016;17:547.

49. Dyrkorn R, Gjelstad S, Espnes KA, et al. Peer academic detailing on use of antibiotics in acute respiratory tract infections. A controlled study in an urban Norwegian out-of-hours service. Scand J Prim Health Care 2016;34:180-5.

50. Delaney BC, Peterson KA, Speedie S, et al. Envisioning a learning health care system: the electronic primary care research network, a case study. Ann Fam Med 2012;10:54-9.

51. Friedman C, Rubin J, Brown J, et al. Toward a science of learning systems: a research agenda for the high-functioning Learning Health System. J Am Med Inform Assoc 2015;22:43-50.
52. Delaney BC, Curcin V, Andreasson A, et al. Translational Medicine and Patient Safety in Europe: TRANSFoRm--Architecture for the Learning Health System in Europe. Biomed Res Int 2015;2015:1-8.

53. Barkhuysen $P$, de Grauw W, Akkermans R, et al. Is the quality of data in an electronic medical record sufficient for assessing the quality of primary care? Journal of the American Medical Informatics Association 2014;21:692-8.

54. van der Bij S, Khan N, Ten Veen P, et al. Improving the quality of EHR recording in primary care: a data quality feedback tool. J Am Med Inform Assoc 2017;24:81-7.

55. Meyer J. Evaluating action research. Age Ageing 2000;29 Suppl 2:8-10.

56. Tonkin-Crine S. Changing th prescibing behaviour of general practitioners: Understanding the acceptability and feasibility of interventions to promote prudent antibiotic use across Europe, 2012. 